\title{
High Spatial Resolution Laser Desorption/Ionization Mass Spectrometry Imaging of Organic Layers in an Organic Light-Emitting Diode
}

\author{
Yuko Tachibana*,1, Yoji Nakajima ${ }^{1}$, Tsuguhide Isemura ${ }^{1}$, Kiyoshi Yamamoto ${ }^{1}$, \\ Takaya Satoh ${ }^{2}$, Jun Aoki ${ }^{3}$, and Michisato Toyoda ${ }^{3}$ \\ ${ }^{1}$ Asahi Glass Co., Ltd., 1150 Hazawa-cho, Kanagawa-ku, Yokohama 221-8755, Japan \\ ${ }^{2}$ JEOL Ltd., 3-1-2 Musashino, Akishima, Tokyo 196-8558, Japan \\ ${ }^{3}$ Project Research Center for Fundamental Sciences, Graduate School of Science, Osaka University, \\ 1-1 Machikaneyama, Toyonaka, Osaka 565-0043, Japan
}

\begin{abstract}
To improve the durability of organic materials in electronic devices, an analytical method that can obtain information about the molecular structure directly from specific areas on a device is desired. For this purpose, laser desorption/ionization mass spectrometry imaging (LDI-MSI) is one of the most promising methods. The high spatial resolution stigmatic LDI-MSI with MULTUM-IMG2 in the direct analysis of organic light-emitting diodes was shown to obtain a detailed mass image of organic material in the degraded area after air exposure. The mass image was observed to have a noticeably improved spatial resolution over typical X-ray photoelectron spectroscopy, generally used technique in analysis of electronic devices. A prospective $\mathrm{m} / \mathrm{z}$ was successfully deduced from the high spatial resolution MSI data. Additionally, mass resolution and accuracy using a spiral-orbit TOF mass spectrometer, SpiralTOF, were also investigated. The monoisotopic mass for the main component, $N, N^{\prime}$-di-1-naphthalenyl- $N, N^{\prime}$-diphenyl-1,1'-biphenyl-4,4'-diamine $(\mathrm{m} / z$ 588), was measured with a mass resolution of approximately 80,000 and a mass error of about $5 \mathrm{mDa}$ using an external calibrant. This high mass resolution and accuracy data successfully deduced a possible elemental composition of partially remained material in the degraded area, $\mathrm{C}_{36} \mathrm{H}_{24}$, which was determined as anthracene, 9-[1,1'-biphenyl]-4-yl-10-(2-naphthalenyl) by combining structural information with highenergy CID data. The high spatial resolution of $1 \mu \mathrm{m}$ in LDI-MSI along with high mass resolution and accuracy could be useful in obtaining molecular structure information directly from specific areas on a device, and is expected to contribute to the evolution of electrical device durability.
\end{abstract}

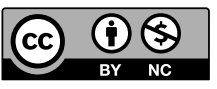

Copyright (c) 2016 Yuko Tachibana, Yoji Nakajima, Tsuguhide Isemura, Kiyoshi Yamamoto, Takaya Satoh, Jun Aoki, and Michisato Toyoda. This is an open access article distributed under the terms of Creative Commons Attribution License, which permits use, distribution, and reproduction in any medium, provided the original work is properly cited and is not used for commercial purposes.

Please cite this article as: Mass Spectrom (Tokyo) 2016; 5(1): A0052

Keywords: laser desorption/ionization mass spectrometry imaging, mass accuracy, MULTUM, SpiralTOF, OLED

(Received July 20, 2016; Accepted November 21, 2016)

\section{INTRODUCTION}

Recently, organic materials have replaced traditional materials used in the construction of various electronic devices. Example applications include organic semiconductors and organic light-emitting diodes (OLED). The use of organic materials in electronic devices has been increasing significantly along with the rapid growth of flexible electronic devices and OLED markets. However, organic ma- terials are subject to degradation when exposed to air and water, which can reduce the functionality of a device. To improve the durability of such materials, it is important to determine the properties of the degraded organic materials and understand the degradation mechanism. A technique is needed that directly analyzes the molecular structures in the degraded areas of a device, and in this regard, molecular imaging shows great promise.

Generally, infrared spectroscopy (IR) and Raman spec-

*Correspondence to: Yuko Tachibana, Asahi Glass Co., Ltd., 1150 Hazawa-cho, Kanagawa-ku, Yokohama 221-8755, Japan, e-mail: yuko-tachibana@agc.com 
troscopy can be used to obtain partial structural information of molecules. However, data obtained by these methods is limited to information about the functional groups, and is often insufficient to identify the organic materials. ${ }^{1)}$

Time-of-flight secondary ion mass spectrometry (TOFSIMS) is an imaging technique that can measure organic materials with sub- $\mu \mathrm{m}$ spatial resolution. ${ }^{2)}$ Due to the recent progress of cluster ion beam technology, fragmentation of molecules has been reduced to some extent, but in many cases, molecular ions do not appear. ${ }^{3)}$ Additionally, despite recent attempts to improve mass accuracy by using better mass-scale calibrants, mass accuracy is often inadequate for determining the identity of unknown materials. ${ }^{4)}$

Matrix-assisted laser desorption/ionization (MALDI) is a soft ionization mass spectrometric technique that results in less molecule fragmentation. When organic layers contain UV absorbers and electrically conductive materials, laser desorption/ionization (LDI) can be directly employed as a soft ionization technique without the use of matrices. ${ }^{5}$ In a report by Moraes et al., degradation of organic material on an OLED device was studied using an LDI-TOF mass spectrometer, but spatial resolution was not discussed and organic material imaging was not applied. ${ }^{6)}$

Mass spectrometry imaging (MSI) is usually performed by scanning a laser beam over the imaging area and obtaining a mass spectrum at each exposure point. In laserscanning imaging, the spatial resolution depends on the radius of laser beam and is around $20 \mu \mathrm{m}$ in usual. In the particular technique using a $\mu \mathrm{m}$-focused laser beam, scanning microprobe MALDI (SMALDI), a high spatial resolution of less than $1 \mu \mathrm{m}$ was reported. ${ }^{7)}$ However, in laserscanning imaging, analysis time from laser beam scanning can be lengthy, sometimes over several hours ${ }^{8)}$ depending on measurement conditions.

In contrast, the stigmatic imaging MALDI-MS incorporating a 2-dimensional detection system reported by Luxembourg et al. demonstrated that the spatial resolution could be independent of the laser beam diameter, and that analysis speed was improved because scanning a small laser beam sequentially over each spot was no longer required. ${ }^{9)}$ A mass image in a $200 \mu \mathrm{m}$ diameter area was measured with a spatial resolution of $4 \mu \mathrm{m}$ over 20 -shots using a laser with a $12 \mathrm{~Hz}$ repetition rate. ${ }^{9)}$

Recently, the stigmatic imaging mass spectrometer 'MULTUM-IMG2'10,11) developed at Osaka University has advanced the stigmatic MSI with a novel ion-extraction method $^{10)}$ and the use of the multi-turn type ion optical system MULTUM II, ${ }^{12)}$ which has achieved high mass resolution by providing an essentially infinite ion flight path by focusing the ions in a figure-eight orbit. The mass image in an area with a diameter of $200 \mu \mathrm{m}$ was measured with a spatial resolution of $1 \mu \mathrm{m}$ and a mass resolution above $10,000 .{ }^{11)}$ It was also reported that using the 'MULTUMIMG,' a previous version of MULTUM-IMG2, the mass image in an area with a diameter of $400 \mu \mathrm{m}$ was measured with 100 -shots using a laser with a repetition rate of $10 \mathrm{~Hz}(10 \mathrm{~s}$ analysis time), and the mass image in a $3.25 \mathrm{~mm} \times 1.5 \mathrm{~mm}$ area was obtained by combining of 78 mass images for detection of dyes on a tissue section. ${ }^{13)}$ This novel MSI technology is expected to be useful in the direct analysis of electrical devices where large-area MSI with high spatial and mass resolution in shorter measurement time helps to obtain detailed information in a specific area while simultaneously gathering information over a wider area.

In addition, the spiral orbit TOF mass spectrometer, SpiralTOF, ${ }^{14)}$ was commercially developed on the basis of MULTUM II technology and improved the mass range limitation in one measurement by overcoming the ion-overtake problem where small, high-speed $m / z$ ions lap the low-speed large $m / z$ ions during flight time.

In this report, we investigated the effectiveness of LDIMSI in the direct analysis of an OLED device. We applied LDI-MSI directly to an OLED device on glass and measured the resulting ions with MULTUM-IMG2. We also looked at detailed mass analysis by SpiralTOF where structural information from tandem mass spectrometry data was combined with high-energy collision-induced dissociation (CID) data to identify an unknown compound.

\section{EXPERIMENTAL}

\section{Instruments of MALDI-TOF mass spectrometer}

The LDI-MSI instrument used in this study was the stigmatic imaging mass spectrometer 'MULTUM-IMG2'10) developed at Osaka University. The third harmonic of a GAIA-II 50T Nd:YAG laser (Rayture Systems, Tokyo, Japan, wavelength $\lambda=355 \mathrm{~nm}$ ) was focused on the target plate to a diameter of approximately $800 \mu \mathrm{m}$. The ions generated from the sample were accelerated at $5 \mathrm{kV}$ by an electric field and passed through a TOF mass analyzer with flight path length of $0.8 \mathrm{~m}$. Ion signal was measured with a 2-dimensional delay-line detector. ${ }^{15)}$ The mass image was obtained over $1.8 \times 10^{5}$ shots using a laser with a repetition rate of $50 \mathrm{~Hz}$.

The LDI-TOF mass spectra of the OLED device was also measured using a JMS-S3000 spiral orbit TOF mass spectrometer, SpiralTOF ${ }^{14)}$ (JEOL Ltd., Tokyo, Japan), with a $17 \mathrm{~m}$ flight path. This system is based on the MULTUM II multi-turn ion optical system using electrostatic sectors. ${ }^{12)}$ A Nd:YLF laser (Newport, CA, USA, wavelength $\lambda=349 \mathrm{~nm}$ ) was used and its radius was fixed to $20 \mu \mathrm{m}$. The OLED device for evaluation was set directly on the target plate in the area where the target plate was sunken $1 \mathrm{~mm}$ in depth. Ions were generated by laser radiation at $1 \mathrm{kHz}$ and accelerated at $20 \mathrm{kV}$. The mass spectrum from the OLED device was externally calibrated using polyethylene glycol (PEG), PEG400 (Sigma-Aldrich, Tokyo, Japan, mass distribution of 200 to $700 \mathrm{Da})$, which was put on an arbitrary area on the target plate, $60 \mathrm{~mm}$ apart from the OLED device. Measurements of the material in the OLED device were carried out without matrix material or cationization agents. Tandem mass spectrometry using high-energy $\mathrm{CID}^{16)}$ was also performed. The selected $20 \mathrm{keV}$ precursor ions were introduced into the collision cell where He gas was used to fragment the ion. The product ions were measured with a reflectron TOF mass spectrometer.

\section{Sample preparation}

An OLED device composed of the following stacked layers on a glass substrate is shown in Fig. 1: aluminum as the cathode layer, lithium fluoride as the electron injection layer, tris(8-hydroxyquinoline) aluminum $\left(\mathrm{Alq}_{3}\right)$ as the electron transport layer, anthracene, 9-[1,1'-biphenyl]-4-yl-10-(2naphthalenyl) as the emitting layer, $N, N^{\prime}$-di-1-naphthalenyl$N, N^{\prime}$-diphenyl-1,1'-biphenyl-4,4'-diamine (NPD), $\mathrm{C}_{44} \mathrm{H}_{32} \mathrm{~N}_{2}$, as the hole transport layer, copper phthalocyanine as the 


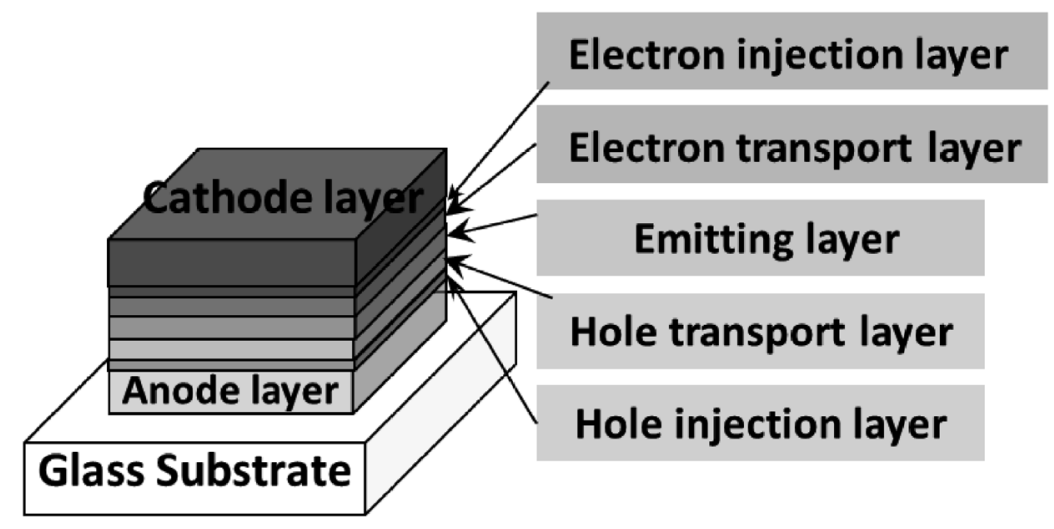

Fig. 1. Schematic of an OLED device composed of stacked layers on glass substrate.

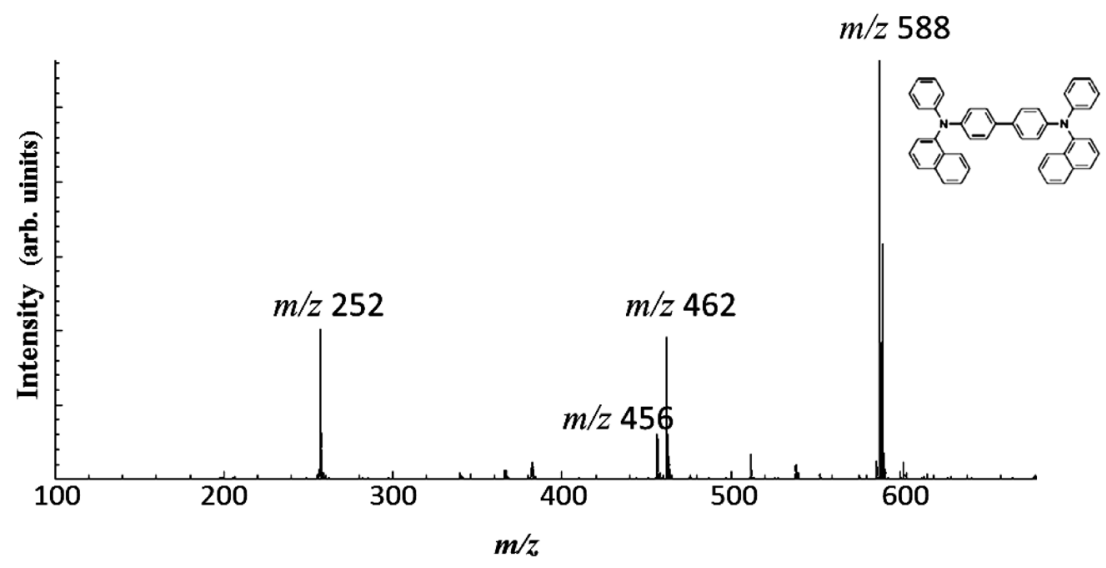

Fig. 2. Integrated MULTUM-IMG2 mass spectrum of the device sample, including the degraded area.

hole injection layer and indium tin oxide as the anode layer. The device was encapsulated under a nitrogen atmosphere with a glass lid and sealed in order to avoid the degradation of the organic layers due to humidity and oxygen in air. The light-emitting area was divided into $200 \mu \mathrm{m}$ squares for each picture cell. For LDI-MSI measurement, the device was removed from encapsulation, and the organic layer was exposed to air by applying adhesive tape to the aluminum cathode top layer and subsequently removing it along with several layers of the device. The degraded area, a whitely colored area observed under the optical microscope, appeared 1 -year after exposure to the air.

\section{RESULTS AND DISCUSSION}

\section{LDI-MSI of the organic materials of the OLED de- vice sample}

LDI-MSI was used to directly measure the OLED device sample. Figure 2 shows a MULTUM-IMG2 integrated mass spectrum of the device sample including the degraded area, a whitely colored area that was observed under optical microscope. A much more intense $\mathrm{m} / z 588$ peak was observed for the molecular ion of NPD. This shows that during sample preparation, the device sample was divided at the interlayer between the electron transport and the emitting layers and that NPD was exposed.
We investigated the peaks shown in Fig. 2 using mass spectrometry imaging. The mass image of $m / z 456$ (Fig. 3a) coincided quite well with the optical microscope image of the degraded area (Fig. 3b). This suggests a possible relationship between the $m / z 456$ compound and the material in the degraded area, which was revealed by exclusive characteristics of MSI. In detail, these images have some differences, which is thought to be caused by the effect of surface morphology or the amount of $\mathrm{m} / \mathrm{z} 456$ material on the scattered reflection of visible light in Fig. 3b. However, in the selected area where other factors such as surface morphology insignificantly affect, the obtained mass image (Fig. 3a) is very similar to the optical microscope image (Fig. 3b), which is thought to be near the previously reported $1 \mu \mathrm{m}$ spatial resolution. ${ }^{11)}$ This is a noticeable improvement compared to more generally used techniques in the analysis of electronic devices, such as X-ray photoelectron spectroscopy (XPS) and IR, which have typical spatial resolutions of $20-200 \mu \mathrm{m}$. Considering that most electrical devices consist of compartments with various sizes that can be as small as several tens of micrometers in width, the stigmatic LDI-MSI could be useful for the direct analysis of partially degraded areas in these devices. 
(a)
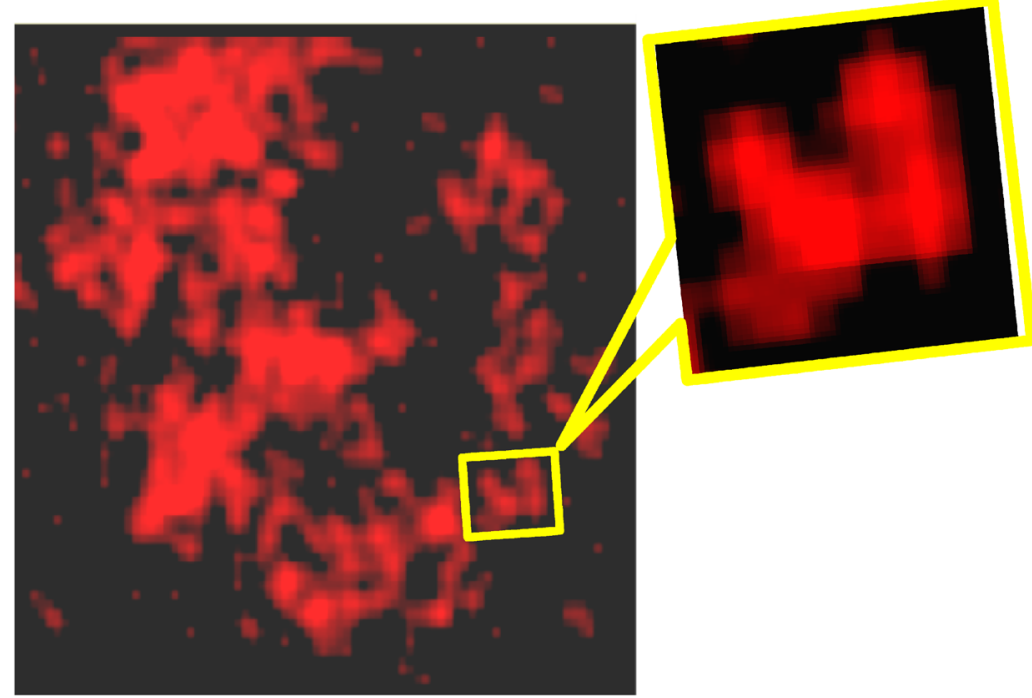

(b)

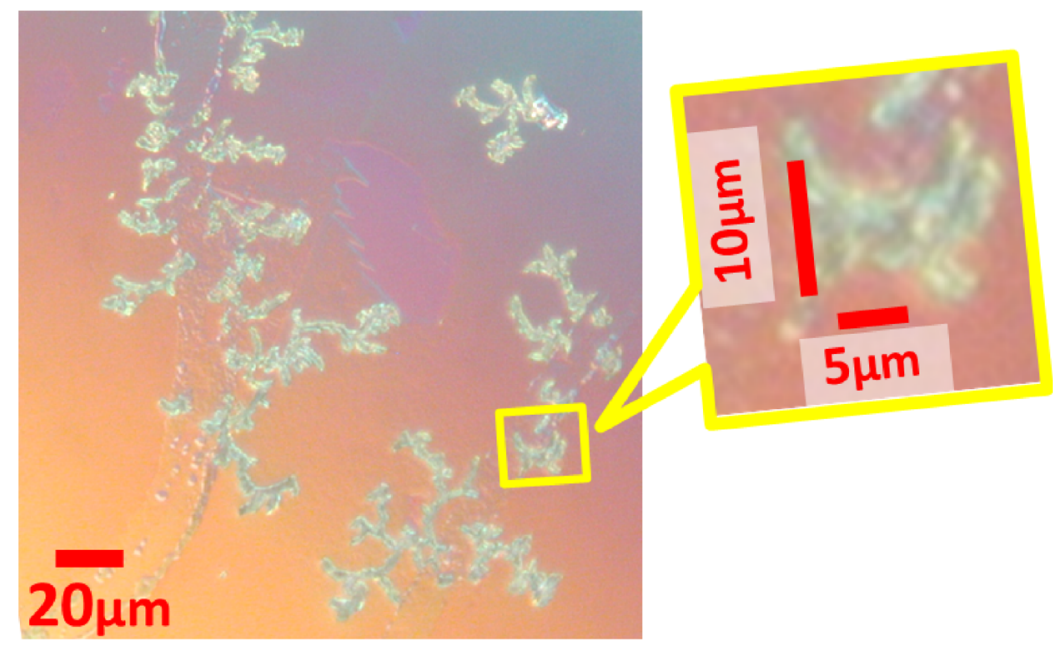

Fig. 3. The mass image of $m / z 456$ by MULTUM-IMG2 (a) and the optical microscope image (b).

LDI-TOF mass spectrometry of the organic materials of the OLED device sample, using SpiralTOF mass spectrometer with high-energy CID

Detailed mass analysis was investigated using a SpiralTOF mass spectrometer. With PEG as an external mass calibrant, the monoisotopic mass for the $\mathrm{m} / \mathrm{z} 588$ peak assigned to NPD was measured to be $\mathrm{m} / z 588.2611$ with mass resolution of about 80,000 . The difference between the measured accurate mass and the calculated exact mass was $5.1 \mathrm{mDa}$, which is sufficiently accurate for this application. We confirmed that high mass accuracy could be obtained in the direct measurement of a device sample on a glass substrate.

Figure 4 shows the mass spectra collected from the normal and the degraded areas. The measured accurate mass of the peak observed at $\mathrm{m} / \mathrm{z} 456$ was $\mathrm{m} / \mathrm{z} 456.1893$. One possible elemental composition calculated from this result is $\mathrm{C}_{36} \mathrm{H}_{24}$ with error within $3 \mathrm{mDa}$.

For tandem mass spectrometry measurements, $m / z 456.19$ was selected as the precursor ion and fragmented using highenergy CID. Results are shown in Fig. 5. As shown by the as- signed peaks in the figure, the candidate material is anthracene, 9-[1,1'-biphenyl]-4-yl-10-(2-naphthalenyl). This compound was used as the emitting layer in the OLED sample and is thought to have partially remained on the NPD layer.

From these results, the degraded area is thought to be the result of crystallization of the anthracene derivative, which is transferred from the adjacent layer and observed to be whitely colored from the scattering of visible light.

These results show that high mass resolution and high mass accuracy of LDI-TOF mass spectrometry is effective for the determination of the organic material by combining structural information with high-energy CID data.

\section{CONCLUSION}

In this paper, we demonstrated the effectiveness of LDIMSI with high spatial resolution for the direct analysis of an electrical device. When combined with structural information obtained from tandem MS measurements with high mass accuracy and resolution, crucial information about the degradation of an OLED device was deduced. 

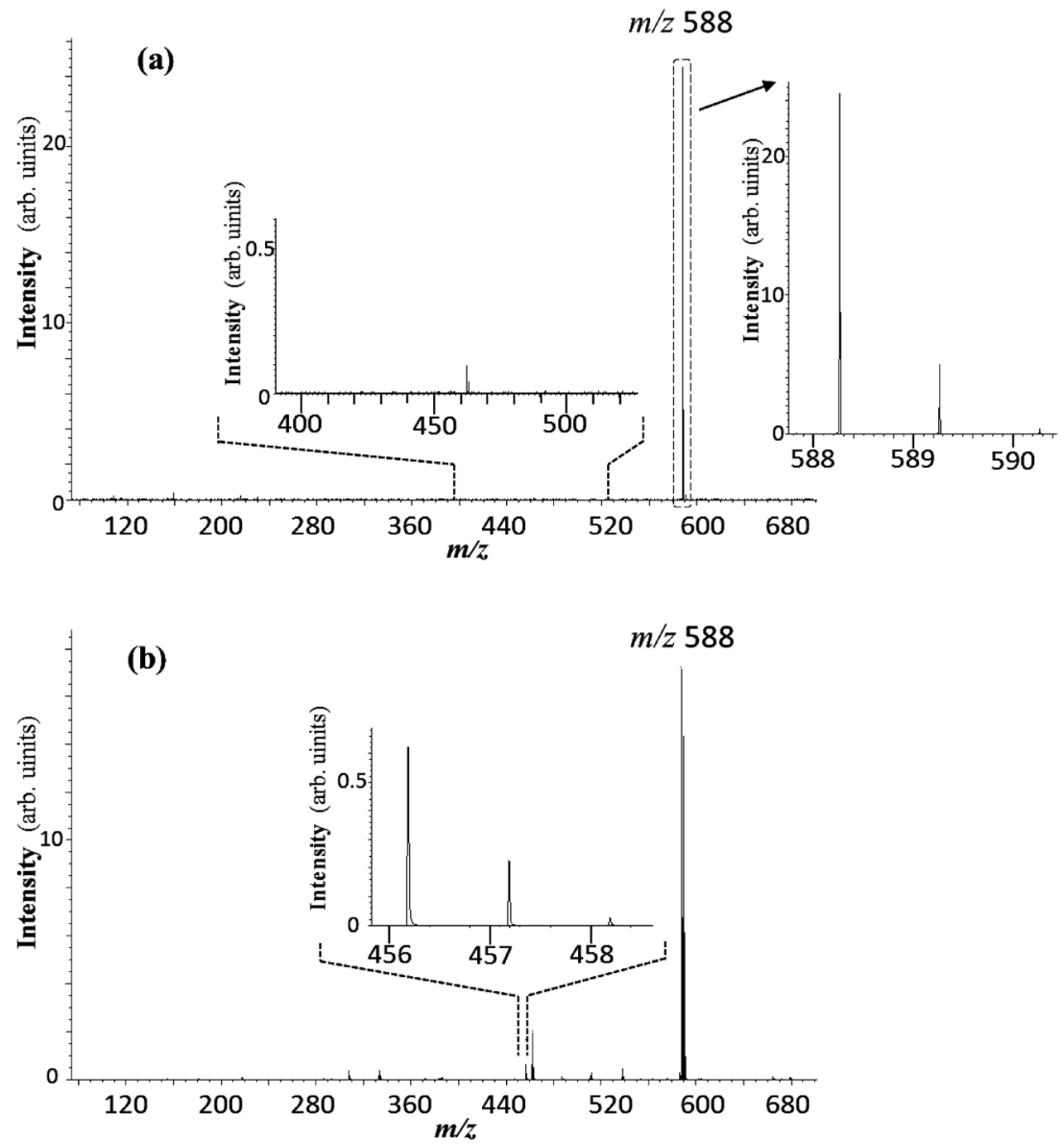

Fig. 4. Mass spectra measured by a Spiral-TOF mass spectrometer for the (a) normal area and (b) degraded area.

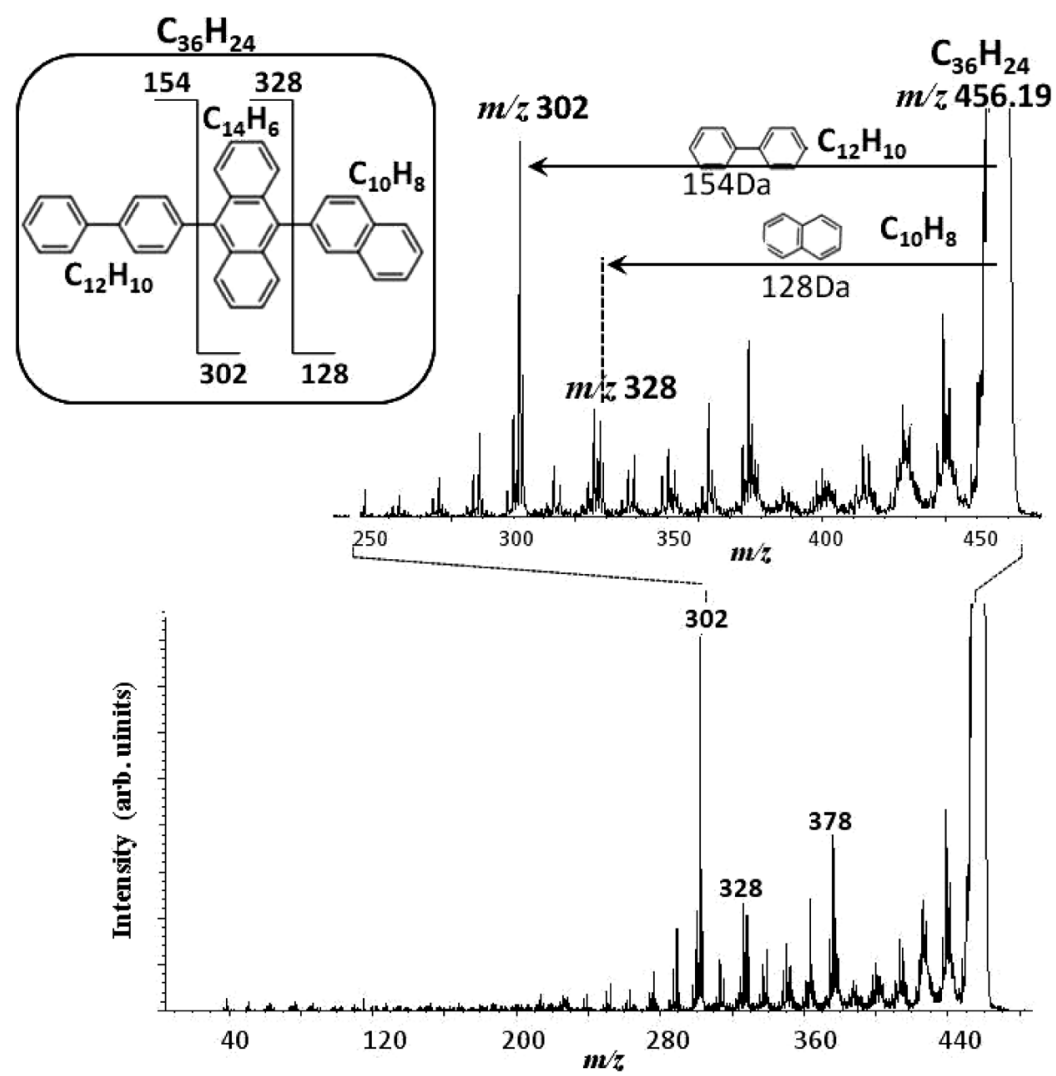

Fig. 5. Product ion spectrum of $m / z 456.19$ using high-energy CID 
The direct analysis of an electrical device with the high spatial resolution stigmatic LDI-MSI instrument MULTUM-IMG2 produced the detailed mass image of the organic material in the device. The mass image was observed to have a noticeably improved spatial resolution over typical XPS and IR, in the selected area where other factors such as surface morphology insignificantly affect. This high spatial resolution MSI data was successfully used to postulate a prospective $m / z$ in the specific area.

We have also shown that high mass resolution and accuracy can be obtained in the direct analysis of electrical device by using an LDI-TOF mass spectrometer with SpiralTOF. The monoisotopic mass for the main component, NPD, was measured with mass resolution of approximately 80,000 with a mass error of approximately $5 \mathrm{mDa}$ after external calibration for a material below $\mathrm{m} / z$ 1,000. This high mass resolution and accuracy was used to calculate a possible molecular composition, and determine the identity of the organic material by combining structural information with tandem mass spectrometry results.

The high spatial resolution LDI-MSI with high mass resolution and accuracy could be helpful in obtaining molecular structure information in specific areas on electrical devices, which is expected to contribute to the evolution of electrical device durability.

\section{Acknowledgements}

The authors wish to thank Dr. Kirk Jensen in Osaka University for helpful technical discussion and comments on this paper.

\section{REFERENCES}

1) I. Razanau, T. Mieno, V. Kazachenko. Thin polymerized $\mathrm{C}_{60}$ coatings deposited in electrostatic field via electron-beam dispersion of fullerite. Thin Solid Films 519: 1285-1292, 2010.

2) K. Norrman, N. B. Larsen, F. C. Krebs. Lifetimes of organic photovoltaics. Combining chemical and physical characterization techniques to study degradation mechanism. Sol. Energy Mater. Sol. Cells 90: 2793-2814, 2006.

3) L. Zhu, F. Li. Direct measurement of bonded film thickness of A20H lubricant. Appl. Phys. A, Mater. Sci. Process. 95: 833-841, 2009.
4) D. Kobayashi, S. Otomo, S. Aoyagi, H. Itoh. Mass-scale calibration of TOF-SIMS spectra using quaternary ammonium ions. Surf. Interface Anal. 46: 229-232, 2014.

5) K. Wolkenstein, J. H. Gross, T. Oeser, H. F. Scholer. Spectroscopic characterization and crystal structure of the 1,2,3,4,5,6-hexahydrophenanthro[1,10,9,8-opqra] perylene. Tetrahedron Lett. 43: 1653-1655, 2002.

6) I. R. Moraes, S. Scholz, B. Lussem, K. Leo. Analysis of chemical degradation mechanism within sky blue phosphorescent organic light emitting diodes by laser-desorption/ionization time-offlight mass spectrometry. Org. Electron. 12: 341-347, 2011.

7) B. Spengler, M. Hubert. Scanning microprobe matrix-assisted laser desorption ionization (SMALDI) mass spectrometry: Instrumentation for sub-micrometer resolved LDI and MALDI surface analysis. Am. Soc. Mass Spectrom. 13: 735-748, 2002.

8) S. Oda-Tamai. Molecular imaging in mouse brain with MALDITOF-MS. J. Yasuda Women's Univ. 40: 389-393, 2012.

9) S. L. Luxembourg, T. H. Mize, L. A. McDonnell, R. M. A. Heeren. High-spatial resolution mass spectrometric imaging of peptide and protein distributions on a surface. Anal. Chem. 76: 53395344,2004

10) J. Aoki, H. Hazama, M. Toyoda. Novel ion extraction method for imaging mass spectrometry. J. Mass Spectrom. Soc. Jpn. 59: 57-61, 2011.

11) J. Aoki, M. Toyoda. Development of stigmatic time-of-flight imaging mass spectrometer. J. Mass Spectrom. Soc. Jpn. 61: 23-33, 2013.

12) M. Toyoda, D. Okumura, M. Ishihara, I. Katakuse. Multi-turn time-of-flight mass spectrometers with electrostatic sectors. J. Mass Spectrom. 38: 1125-1142, 2003.

13) H. Hazama, H. Yoshimura, J. Aoki, H. Nagao, M. Toyoda, K. Masuda, K. Fujii, T. Tashima, Y. Naito, K. Awazu. Development of a stigmatic mass microscope using laser desorption/ionization and a multi-turn time-of-flight mass spectrometer. J. Biomed. Opt. 16: 046007, 2011.

14) T. Satoh, T. Sato, J. Tamura. Development of a high-performance MALDI-TOF mass spectrometer utilizing a spiral ion trajectory. J. Am. Soc. Mass Spectrom. 18: 1318-1323, 2007.

15) O. Jagutzki, V. Mergel, K. U. Pfleger, L. Spielberger, U. Meyer, R. Doerner, H. W. S. Boecking. Fast position and time-resolved read-out of micro-channelplates with the delay-line technique for single-particle and photon-detection. Proceedings of SPIE 3438. Imaging Spectrometry IV: 322-333, 1998.

16) T. Satoh, T. Sato, A. Kubo, J. Tamura. Tandem time-of-flight mass spectrometer with high precursor ion selectivity employing spiral ion trajectory and improved offset parabolic reflectron. $J$. Am. Soc. Mass Spectrom. 22: 797-803, 2011. 